\title{
Doença arterial coronariana e suporte familiar em idosos
}

\author{
Coronary artery disease and family support in older adults \\ Enfermedad arterial coronaria y apoyo familiar en ancianos
}

\author{
Lauana Aparecida Teodoro Jacintor ; Álvaro da Silva Santos" ; Marina Aleixo Diniz ${ }^{\text {III. }}$ \\ Larissa Carvalho Silva ${ }^{I V}$; Fabiola Silva de Sousa Pedrosav; Juliana Barbosa Arduini ${ }^{V I}$
}

\begin{abstract}
RESUMO: Estudo quantitativo, com aplicação de questionário através de entrevista, realizado em 2010, com o objetivo de analisar fatores de risco para doença arterial coronária e a relação de suporte familiar em idosos cadastrados na Estratégia Saúde da Família de Uberaba/MG. Foram pesquisados 853 idosos. A faixa etária prevalente foi de 60 a 79 anos $(88,6 \%)$; mulheres $(63,7 \%)$; aposentados $(81,1 \%)$; com até dois salários mínimos (78,1\%); hipertensão arterial (65\%); sedentarismo (59\%); diabetes mellitus (28\%); tabagismo (21\%); etilismo (12,1\%); dislipidemia (4,8\%); convívio familiar ótimo $(49,7 \%)$, e bom $(42 \%)$; tem acompanhamento familiar do estado de saúde $(81,7)$; mas não há cooperação familiar no exercício físico $(86 \%)$, medicação $(66,7 \%)$, recursos financeiros $(61,4 \%)$ e alimentação $(60,5 \%)$. Conclui-se neste estudo que profissionais de saúde devem estimular o suporte familiar, e juntamente com o Estado, atuar criando políticas e estratégias na redução dos fatores de risco para doenças cardiovasculares.
\end{abstract}

Palavras-Chave: Doenças cardiovasculares; fatores de risco; idoso; relações familiares.

ABSTRACT: This quantitative study, based on questionnaires applied at interview, was conducted in 2010 to analyze risk factors for coronary artery disease and the relationship of family support in 853 older adults registered with the family health program in Uberaba, Minas Gerais, Brazil. The group was prevalently aged 60 to 79 years $(88.6 \%)$; women $(63.7 \%)$ and retirees $(81.1 \%)$; with income up to 2 minimum wages $(78.1 \%)$; arterial hypertension $(65 \%)$; sedentary lifestyle $(59 \%)$; diabetes mellitus (28\%); smoking (21\%); alcohol (12.1\%); dyslipidemia (4.8\%); family relations excellent $(49.7 \%)$ and good $(42 \%)$; family monitors state of health (81.7); but does not cooperate with physical exercise $(86 \%)$, medication $(66.7 \%)$, financial resources $(61.4 \%)$ and food $(60.5 \%)$. It was concluded that health professionals should encourage family support, and together with the State, act to formulate policies and strategies to reduce risk factors for cardiovascular diseases.

Keywords: Cardiovascular diseases; risk factors; older adults; family relations.

RESUMEN: Estudio cuantitativo, con cuestionarios a través de entrevista, realizado en 2010, con el objetivo de analizar los factores de riesgo para enfermedad arterial coronaria y la relación del apoyo familiar en ancianos inscritos en la estrategia salud de la familia de Uberaba / MG, Brasil. Fueron encuestados 853 ancianos. El grupo etario predominante fue de 60 a 79 años $(88,6 \%)$ mujeres $(63,7 \%)$; jubilados $(81,1 \%)$; con hasta dos salarios mínimos $(78,1 \%)$; hipertensión arterial $(65 \%)$; sedentarios (59\%); diabetes mellitus (28\%); fumantes $(21 \%)$; y alcoholismo $(12,1 \%)$; dislipidemia $(4,8 \%)$; convivencia familiar ótima $(49,7 \%)$ y buena $(42 \%)$; tiene monitoreo familiar del estado de salud $(81,7)$; pero no hay ninguna cooperación en ejercicio físico $(86 \%)$, medicamentos $(66,7 \%)$, recursos financieros $(61,4 \%)$ y alimentos $(60,5 \%)$. Este estudio concluyó que profesionales de salud deben estimular el apoyo familiar y, junto con el Estado, actuar creando políticas y estrategias en la reducción de factores de riesgo para enfermedades cardiovasculares.

Palabras Clave: Enfermedades cardiovasculares; factores de riesgo; anciano; relaciones familiares.

\section{INTRODUÇÃO}

A questão do envelhecimento pode ser entendida como uma condição de grande impacto social, pois as doenças e agravos não transmissíveis (DANT) oneram o sistema social e desqualificam a vida de seu portador devido ao gasto em medicamentos, complicações e dificuldades no cuidado. Porém, é um ganho sócio-demográfico pela ampliação da expectativa de vida.

\footnotetext{
Enfermeira. Especialista em Saúde Pública e da Família. Mestre em Atenção à Saúde. Enfermeira da Secretaria de Estado da Defesa Social de Minas Gerais. Uberaba, Minas Gerais, Brasil. E-mail: lauana_jacinto@yahoo.com.br.

IIEnfermeiro. Doutor em Ciências Sociais. Professor Adjunto III no Departamento de Enfermagem em Educação e Saúde Comunitária e do Programa de Pós-Graduação em Atenção à Saúde (Mestrado e Doutorado) da Universidade Federal do Triângulo Mineiro. Uberaba, Minas Gerais, Brasil. Lider do Grupo de Pesquisa Ciclos de Vida, Família e Saúde no Contexto Social. E-mail: alvaroenf@hotmail.com.

IIIEnfermeira. Mestre em Atenção à Saúde. Doutoranda do Programa de Pós-Graduação em Enfermagem Fundamental da Escola de Enfermagem de Ribeirão Preto. São Paulo, Brasil. E-mail: mafmtm@yahoo.com.br.

IVEnfermeira. Especialista em Urgência e Emergência. E-mail: lissacarvalhos@hotmail.com.

vEnfermeira da Secretaria Municipal de Saúde de Conceição das Alagoas. Minas Gerais. E-mail: fafasousa2003@hotmail.com.

${ }^{\mathrm{VI} E n f e r m e i r a . ~ E s p e c i a l i s t a ~ e m ~ S a u ́ d e ~ P u ́ b l i c a ~ e ~ d a ~ F a m i ́ l i a, ~ H o s p i t a l ~ d e ~ C l i ́ n i c a s ~ d a ~ U n i v e r s i d a d e ~ F e d e r a l ~ d o ~ T r i a ̂ n g u l o ~ M i n e i r o . ~ U b e r a b a, ~ M i n a s ~ G e r a i s, ~}$ Brasil.E-mail: julianabarduini@yahoo.com.br.

VIITrabalho financiado pela Fundação de Amparo a Pesquisa de Minas Gerais.
} 
A população brasileira está envelhecendo de forma acentuada. De acordo com dados do Instituto Brasileiro de Geografia e Estatística (IBGE) ${ }^{1}$ a expectativa de vida atual é de 74,6 anos. A Organização Mundial de Saúde (OMS) classifica o indivíduo como idoso aquele de 65 anos ou mais de idade, em países desenvolvidos, e 60 anos ou mais em países subdesenvolvidos ${ }^{2,3}$.

O envelhecimento deve ser compreendido como processo não patológico, com diminuição progressiva da reserva funcional. Entretanto, em condições de sobrecarga, como na vigência de doenças cardiovasculares e estresse emocional, podem-se instalar quadros patológicos que exigem assistência, caracterizando a senilidade ${ }^{3,4}$. Essa condição de debilidade física ou intelectual pode ser evitada quanto menor for a exposição aos fatores de risco para as doenças cardiovasculares ${ }^{4}$. Dado a isto, a atenção ao idoso deve ser baseado nas alterações próprias do envelhecimento, buscando um envelhecimento saudável e qualidade de vida ${ }^{5,6}$.

As doenças cardiovasculares (DCV) representam um grande impacto em termos de mortalidade e morbidade para a sociedade brasileira, tendo sido responsáveis por 28\% dos óbitos no período de 1996 a 2011. As DCV lideram os índices de morbidade e mortalidade no Brasil e no mundo, sendo a doença arterial coronariana (DAC) a causa de um grande número de mortes e de gastos em assistência médica ${ }^{7,8}$.

O suporte familiar pode ser definido como recurso primário de socialização na qual padrões de comportamento, linguagem, valores, crenças e costumes são transmitidos e as necessidades psicológicas e fisiológicas básicas são atendidas. É um processo recíproco entre os membros da família, que gera efeitos tanto para quem recebe, como para quem oferece 9 . O idoso pode necessitar de suporte familiar independente da presença de doenças, de várias formas. No caso de idoso com DAC, pode ser necessário suporte familiar tanto em aspectos fisiológicos, como emocionais.

Assim, teve-se como objetivo deste estudo: analisar fatores de risco para doença arterial coronária e a relação de suporte familiar em idosos cadastrados na Estratégia Saúde da Família de Uberaba/MG.

\section{REVISÃo DE LITERATURA}

O envelhecimento populacional esta entre as principais preocupações em um país que é visto em todo o mundo como sinônimo de juventude. Mas isso vem mudando nas últimas décadas: o Brasil embarcou em um processo de desenvolvimento que o está levando a atingir índices sociais e demográficos de Primeiro Mundo, embora com sistemas e instituições herdadas de outro contexto ${ }^{10}$.

No Brasil, segundo a Síntese de Indicadores Sociais de 2011, realizado pelo IBGE, as doenças do aparelho circulatório atingiram $37,5 \%$ das mortes e são a principal causa de mortes no país entre os idosos ${ }^{11}$. O fumo, a obesidade, o Diabetes Mellitus (DM), a hipertensão arterial, os níveis elevados de colesterol, a história familiar de DAC e o sedentarismo aumentam o risco da doença ${ }^{12,13}$. Por sua vez, fatores não modificáveis são aqueles nos quais não se pode exercer influência, não podendo ser excluídos, compreendendo: etnia, sexo, idade e a história familiar ${ }^{5}$.

Estilo de vida e estresse emocional são fatores de risco para doenças cardiovasculares e têm merecido destaque na literatura. Estudos mostraram uma maior incidência de eventos cardiovasculares na população com sintomas depressivos ou baixos escores de indicadores de qualidade de vida. Pacientes com DAC já instalada apresentam qualidade de vida prejudicada e nível elevado de estresse, impostos pela própria condição de doença, limitação física e risco de morte, fechando um círculo vicioso que intensifica a progressão da doençą ${ }^{14}$.

$\mathrm{Na}$ DAC e nas DCV, na perspectiva preventiva ou de tratamento, é importante a participação da família, principalmente no suporte ao idoso. Frequentemente tais idosos tendem a consumir vários medicamentos e dietas especiais, a receber prescrição de atividade física e, ainda, de controle de estresse, condições que em geral necessitam de suporte da família.

As mudanças de status das mulheres (principais cuidadoras) e dos valores sociais e familiares continuarão afetando a disponibilidade de apoio familiar para os idosos ${ }^{11}$. A prioridade na atenção à saúde dos idosos deve voltar-se para as estratégias que possibilitem a vida mais saudável, a monitorização de indicadores capazes de avaliar a morbidade, o impacto da doença e/ou incapacidade na qualidade de vida dos idosos e de suas famílias ${ }^{15}$.

A busca do envelhecimento saudável é um desafio da atualidade, e se reveste de maior importância no sentido de oferecer aos idosos maior autonomia, qualidade de vida, para além do cuidado de doenças preexistentes. Nessas ações, os profissionais devem estar atentos não só às alterações físicas e fisiológicas, advindas do processo de envelhecimento, mas também às alterações na dinâmica sociofamiliar, as quais são determinantes na capacidade funcional e, consequentemente, no envelhecimento saudável ${ }^{16}$. Desse modo, os profissionais de saúde devem investigar os fatores que têm impactado negativamente a qualidade de vida dos idosos, buscando possíveis soluções juntamente com seus familiares ${ }^{17}$.

Dimensionar estilos de vida não saudáveis (consumo de gorduras, obesidade, estresse não controlado, sedentarismo, entre outros) e predisponentes às DAC, e as relações de suporte familiar, se justificam por si só na medida em que podem oferecer dados para intervenções em idosos em risco ou acometidos nesse agravo. 


\section{Metodologia}

Este é um estudo de caráter quantitativo e descritivo, que é a descrição de certas características quantitativas de populações como um todo ${ }^{18}$.

Este tipo de investigação se aplica às intenções deste estudo, uma vez que apresentará características para riscos de doença arterial coronariana e as relações de apoio familiar na população idosa de Uberaba, além do que fornecerá dados para outras investigações e poderá dar sustentação às políticas públicas voltadas para a população idosa, com vistas à redução dos riscos cardiovasculares.

A cidade de Uberaba/MG tinha uma população, em 2009, de 296.261 habitantes, das quais 10,2 \% eram pessoas com 60 anos ou mais. A pesquisa foi realizada com usuários de idade maior ou igual a 60 anos, adscritos nas unidades de saúde da família (USF), e que estivessem em atendimento no período do estudo.

A coleta de dados se deu no período de junho a agosto de 2010. Os entrevistados foram selecionados nas unidades ou nas suas residências, a cada cinco idosos na listagem dos agentes comunitários de saúde (ACS), após orientação sobre condições da pesquisa com a assinatura do Termo de Consentimento Livre e Esclarecido. A amostra então foi por conveniência. A coleta de dados se deu pelos pesquisadores ou ACS treinados, nas residências ou sala reservada da USF.

Os critérios de inclusão foram: ter 60 ou mais anos, cadastrados nas USF do município e ser sorteado sem reposição a cada cinco idosos na listagem dos ACS. Como critério de exclusão foram previstos: idosos que se negassem a participar do estudo, não ser sorteado, com pontuação igual ou menor que 12 no Mini Exame do Estado Mental (MEEM), ou se recusarem ou não estarem nas suas residências no momento da entrevista. A amostra se compôs de 853 idosos. O MEEM é um instrumento validado que avalia estado cognitivo e considera declínio cognitivo quando o idoso obtém pontuação igual ou inferior a 12 pontos $^{19}$.

Após se obter pontuação maior ou igual a 13 no MEEM, um questionário construído pelos pesquisadores, com 39 questões fechadas, foi aplicado aos idosos que responderam aos critérios de inclusão. Para este artigo foram apresentadas as seguintes variáveis: sociodemográficas - faixa etária, sexo, aposentadoria, renda familiar, cor e situação conjugal; fatores de risco para DAC - tabagismo, alcoolismo, realização de atividade física, agravos à saúde; suporte familiar - número de moradores no domicilio, números de filhos, números de filhos que moram no mesmo domicilio, suporte familiar na alimentação/ medicação/atividade física/recursos financeiros, convívio familiar e acompanhamento familiar sobre saúde.

O projeto foi submetido ao Comitê de Ética em Pesquisa da Universidade Federal do Triângulo Mineiro e aprovado sob o parecer $\mathrm{n}^{\circ} 1527$.
Os dados foram analisados pelo Statiscal Package for the Social Science (SPSS) 16 e os dados numéricos apresentados em valores absolutos e percentuais. As categorias foram analisadas através da distribuição de frequência simples. Os resultados foram dispostos em tabelas para melhor compreensão dos mesmos.

\section{Resultados e Discussão}

Participaram deste estudo 853 idosos cadastrados nas equipes de saúde da família pertencentes aos três distritos sanitários, da cidade de Uberaba, Minas Gerais. Entre os participantes da pesquisa, $543(63,7 \%)$ eram do sexo feminino e $310(36,3 \%)$ do sexo masculino, dos quais $539(63,2 \%)$ eram da cor branca, 172(20,2\%) - negra, 129(15,1\%) - parda e $13(1,5 \%)$ - amarela. A faixa etária mais prevalente foi entre 60 a 69 anos - $458(53,7 \%)$, seguida de 70 a 79 anos - 298(34,9\%). Quanto à situação conjugal, 434 (50,9\%) afirmaram viver com parceiro e 419(49,1\%) não. Em relação a ter uma ocupação remunerada, somente $128(15 \%)$ relataram trabalhar e $725(85 \%)$ não são ocupados. A maioria - 692(81,1\%) - referiu ser aposentada, sendo que 334(39,2\%) viviam com renda familiar de um salário mínimo e 332(38,9\%) com dois salários, apenas 24(2,8\%) com cinco salários ou mais, de acordo com a Tabela 1.

TABELA 1: Caracteristicas sciodemograficas dos idosos pesquisados de Uberaba-MG, 2010. $(\mathrm{N}=853)$

\begin{tabular}{|c|c|c|}
\hline Variáveis & $f$ & $\%$ \\
\hline \multicolumn{3}{|l|}{ Sexo } \\
\hline Masculino & 309 & 36,3 \\
\hline Feminino & 543 & 63,7 \\
\hline \multicolumn{3}{|l|}{ Faixa etária (em anos) } \\
\hline $601-69$ & 458 & 53,7 \\
\hline $701-79$ & 298 & 34,9 \\
\hline $801-89$ & 88 & 10,3 \\
\hline 90 ou mais & 9 & 1,1 \\
\hline \multicolumn{3}{|l|}{ Situação conjugal } \\
\hline Vive com parceiro & 434 & 50,9 \\
\hline Não vive com parceiro & 419 & 49,1 \\
\hline \multicolumn{3}{|l|}{ Aposentados } \\
\hline Não & 161 & 18,9 \\
\hline Sim & 692 & 81,1 \\
\hline \multicolumn{3}{|l|}{ Renda familiar } \\
\hline 1 salário & 334 & 39,2 \\
\hline 2 salários & 332 & 38,9 \\
\hline 3 salários & 118 & 13,8 \\
\hline 4 salários & 45 & 5,3 \\
\hline 5 salários ou mais & 24 & 2,8 \\
\hline \multicolumn{3}{|l|}{ Cor } \\
\hline Branca & 539 & 63,2 \\
\hline Preta & 172 & 20,2 \\
\hline Parda & 129 & 15,1 \\
\hline Amarela & 13 & 1,5 \\
\hline
\end{tabular}


Baixa renda familiar, viver sem parceiro e ser mulher parecem condições predisponentes à maior dependência de suporte familiar. Alguns estudos de perfil de idosos têm mostrado dados similares aos aqui apresentados ${ }^{20,21}$. Um estudo de Pelotas, com 991 idosos, mostrou maior número de mulheres, sem parceiros e com associação significativa para inatividade física e incapacidade funcional ${ }^{20}$. Estudo realizado em Guarapuava, com 359 idosos, apontou baixa renda, predominância de mulheres e vivendo com parceiro ${ }^{21}$.

A baixa renda limita o acesso a bens de serviços e de consumo, como alimentação e moradia adequadas. Quanto à ocupação, pesquisas demonstram que aposentadorias e pensões constituem a principal fonte de renda dos idosos brasileiros ${ }^{22}$.

Em relação ao estilo de vida que pode interferir na saúde dos idosos e favorecer a DAC, observou-se que a maioria - 674(79\%) - refere não fumar e 179(21\%) fumam, dos quais $82(46 \%)$ fumam de 1 a 9 cigarros por dia e 33(18,8\%) 20 ou mais. O consumo de álcool foi referido por $103(12,1 \%)$, sendo que destes $29(28,15 \%)$ consomem cinco copos por semana e $11(10,7 \%)$ ingerem cinco copos ou mais por dia. A maioria - 503(59\%) - não realiza nenhuma atividade física (AF) e 350(41\%) informaram praticá-la; a atividade mais praticada é a caminhada - 305 (87,1\%). Dos principais agravos, a hipertensão arterial aparece em 441(65\%), o DM em $190(28 \%)$ e as dislipidemias em 32(4,8\%), de acordo com a Tabela 2.

O estilo de vida pode interferir na saúde dos idosos e levar a uma possível DAC. Verificou-se a hipertensão arterial, o sedentarismo, o DM, o tabagismo e o alcoolismo, seguidos de dislipidemia, como fatores de risco importantes no grupo pesquisado. Estudos sobre perfil de idosos mostraram como fatores de risco: hipertensão arterial e alcoolismo $0^{5,23,24}$.

A hipertensão arterial é importante condição para a DAC. Estudo sobre a prevalência e fatores de risco na associação entre DAC e aneurisma de aorta refere que, comparando pacientes com e sem DAC, as situações de risco mais frequentes estavam no grupo de pacientes portadores de coronariopatia, entretanto apenas a hipertensão arterial e a dislipidemia foram estatisticamente significantes ${ }^{25}$.

Outro fato relevante consiste na não realização de AF pelos idosos entrevistados. Estudos demonstram que há uma diminuição do nível de atividade relacionada ao aumento da idade, em desacordo com a teoria da atividade que argumenta sobre a necessidade do idoso de manter os níveis de atividade apesar das perdas associadas ao envelhecimento ${ }^{26}$. Um estudo com idosos em Síndrome Coronariana Aguda (SCA) mostrou o sedentarismo como maior fator de risco ${ }^{27}$.

O Diabetes Mellitus aparece como terceiro fator neste estudo. Do mesmo modo na SCA, observou-se num estudo a DM como importante fator de risco ${ }^{27}$, bem como em outro estudo para fatores de risco em
DAC5. O DM está crescendo rapidamente com o envelhecimento populacional e o controle metabólico deficiente se constitui fator de risco para o desenvolvimento de complicações microvasculares e macrovasculares, que aumentam substancialmente a morbimortalidade associada a essas doenças ${ }^{28}$. Estudo sobre DAC e DM demonstrou que em pacientes com DM tipo 1 tem na DAC a maior causa de morte, pela qual o risco de mortalidade aumenta de 4 a 9 vezes em homens e 4 a 29 vezes em mulheres ${ }^{29}$.

Ao considerar que $179(21 \%)$ dos pesquisados referem tabagismo, é notável que estejam sob maior risco de desenvolver doenças relacionadas ao cigarro, porque tendem à exposições mais longas e mais intensas ao tabaco, se comparados à população mais jovem $^{24}$. O tabaco além de causar alterações cardíacas pode levar a outras condições patológicas, hábito modificável que merece intervenção em idosos.

O etilismo aparece como quinto fator importante. Sabe-se que o álcool pode levar a uma série de condições patológicas. Um estudo mostrou que mais de $10 \%$ eram etilistas, e tal dependência era

TABELA 2: Hábitos de vida e doenças referidas dos idosos pesquisados. Uberaba-MG, 2010. $(\mathrm{N}=853)$

\begin{tabular}{|c|c|c|}
\hline Variáveis & $f$ & $\%$ \\
\hline \multicolumn{3}{|l|}{ Tabagismo } \\
\hline Sim & 179 & 21 \\
\hline Não & 674 & 79 \\
\hline \multicolumn{3}{|l|}{ Quantidade de cigarro } \\
\hline 1l- 9 cigarros / dia & 82 & 46 \\
\hline 10 l- 19 cigarros / dia & 63 & 35,2 \\
\hline 20 cigarros ou mais / dia & 33 & 18,8 \\
\hline \multicolumn{3}{|l|}{ Bebidas alcoólicas } \\
\hline Sim & 103 & 12,1 \\
\hline Não & 750 & 87,9 \\
\hline \multicolumn{3}{|l|}{ Consumo de álcool } \\
\hline 1 I- 4 copos / dia & 22 & 21,3 \\
\hline 5 copos ou mais / dia & 11 & 10,7 \\
\hline 1 I- 4 copos / semana & 41 & 39,8 \\
\hline 5 copos ou mais / semana & 29 & 28,2 \\
\hline \multicolumn{3}{|l|}{ Atividade física } \\
\hline Sim & 350 & 41 \\
\hline Não & 503 & 59 \\
\hline \multicolumn{3}{|l|}{ Quais atividades físicas } \\
\hline Caminhada & 305 & 87,1 \\
\hline Hidroginástica & 52 & 14,9 \\
\hline Ginástica & 25 & 7,1 \\
\hline Natação & 8 & 2,2 \\
\hline Dança & 7 & 2 \\
\hline \multicolumn{3}{|l|}{ Doenças referidas } \\
\hline Hipertensão arterial sistêmica & 441 & 65 \\
\hline Diabetes Mellitus & 190 & 28 \\
\hline Dislipidemias & 32 & 4,8 \\
\hline Problema músculoesquelético & 9 & 1,3 \\
\hline Problemas neurológicos & 6 & 0,8 \\
\hline Problemas gastrointestinais & 1 & 0,1 \\
\hline
\end{tabular}


mais presente em homens ${ }^{30}$. Esta é uma condição importante tanto como fator predisponente ao DAC, como a sua piora, além do que torna o cuidado ao idoso mais complexo.

Quanto ao número de pessoas que habitam o domicílio, 305(35,8\%) vivem com duas pessoas, $134(15,7 \%)$ vivem sozinhos e $118(13,8 \%)$ com cinco ou mais pessoas. Em relação à estrutura familiar, observou-se que a maioria - 812(95,2\%) - tem filhos, com prevalência de 292(34,2\%) com cinco ou mais. Dos que relaram ter filhos, $379(46,6 \%)$ moram com eles.

Ao se questionar sobre quem cuidaria do idoso no caso de alguma enfermidade, $437(51,3 \%)$ referiram ser os filhos, $296(34,7 \%)$ apontaram o cônjuge e $27(3,2 \%)$ responderam que ninguém cuidaria deles.

No que diz respeito ao suporte familiar e mais especificamente ao convívio familiar destinado aos idosos, observou-se que 424(49,7\%) relatam ser ótimo, $358(42 \%)$ - bom, 53(6,2\%) - regular e 18(2,1\%) - ruim. A maioria - $697(81,7 \%)$ - refere que os familiares acompanham o seu estado de saúde. Quanto à cooperação dos familiares, a maioria não a recebe, realizando sozinhos: $734(86 \%)$ - exercício físico, $569(66,7)$ - medicação e 516(60,5\%) - alimentação, conforme a Tabela 3.

Quanto ao tipo de suporte, a maioria refere que os familiares tem ótimo e bom convívio, além do que acompanham o seu estado de saúde, mas não cooperam quanto aos exercícios físicos, medicação, recursos financeiros e alimentação.

Verificou-se que é expressivo o número de idosos que mora só e alguns deles relataram que em situação adversa ninguém cuidaria dele, embora a maioria tenha filhos e um pouco mais da metade viva com parceiro(a), ou até mesmo o filho(a).

TABELA 3: Suporte familiar oferecido aos idosos pesquisados . Uberaba-MG, 2010.

\begin{tabular}{lccc}
\hline \multicolumn{1}{c}{ Variáveis } & $\mathrm{f}$ & $\%$ \\
\hline Convívio familiar & & & \\
$\quad$ Ótimo & & 424 & 49,7 \\
$\quad$ Bom & & 558 & 42 \\
$\quad$ Regular & 18 & 6,2 \\
$\quad$ Ruim & & 2,1 \\
Acompanhamento de saúde & 697 & 81,7 \\
$\quad$ Sim & & 156 & 18,3 \\
$\quad$ Não & & & \\
Cooperação familiar & Sim & 337 & 39,5 \\
$\quad$ Alimentação & Não & 516 & 60,5 \\
& Sim & 329 & 38,6 \\
$\quad$ Recursos Financeiros & Não & 524 & 61,4 \\
$\quad$ Mim & 284 & 33,3 \\
$\quad$ Medicação & Não & 569 & 66,7 \\
$\quad$ Exercício físico & Sim & 119 & 14 \\
$\quad$ Não & 734 & 86 \\
\hline
\end{tabular}

Pesquisa sobre as necessidades de saúde dos idosos revela que os cuidados em domicilio são prestados por familiares, principalmente as filhas. Este estudo cita a Política Nacional da Pessoa Idosa, a qual ressalta que a família deve ser responsável pelo cuidado dos idosos em relação ao atendimento as suas necessidades. Se o cônjuge não pode assumir o cuidado, o papel é assumido pelos filhos. Assim, cabe ao profissional de saúde que assiste ao idoso ter uma visão mais ampla do processo de envelhecimento e da importância da rede de suporte familiar aos idosos ${ }^{31}$.

Outro estudo aponta que os familiares cuidadores são todas mulheres, sem formação para cuidar dos idosos, que referiram não ter recebido instrução formal de profissionais da saúde para a realização de cuidados, realizando-os a partir de suas experiências prévias ${ }^{32}$. Isso reforça ainda mais a importância do profissional de saúde no cuidado ao idoso e no suporte à família.

Estudo realizado com idosos de uma UBS, no Município de Fortaleza-CE, mostrou que o percentual dos que moram sozinhos é significativo, 11,7\%; além disso, 44,9\% moravam em domicilio multigeracional, prevalecendo os domicílios com três gerações (presença de filhos e netos). Idosos que residem em domicílios multigeracionais tendem a ser mais pobres, com maior dependência, podendo representar não uma opção cultural, mas um arranjo para garantir a sobrevivência dos membros da família ${ }^{33}$.

A cooperação familiar nos quesitos exercício físico (EF) e medicação mostraram-se baixos e revelam a necessidade de atenção aos idosos pesquisados, considerando que o EF é necessário à preservação da autonomia e funcionalidade, entre outros; e, na medicação, considerando ser este grupo consumidor da polifarmácia, erros de uso ou de compreensão de prescrição pode influenciar na qualidade de vida do idoso. Um estudo de revisão verificou, em várias pesquisas, que o exercício físico tem se mostrado efetivo na prevenção e controle da hipertensão arterial e cardiopatias ${ }^{34}$. No tocante ao uso de medicações, uma pesquisa mostrou que os idosos veem a orientação médica incompleta e a necessidade de apoio familiar, além da cooperação no uso de polifarmácia e suas consequências ${ }^{35}$. $\mathrm{O}$ apoio de recursos familiares, inclusive na alimentação em qualidade, e quantidade abrange o monitoramento do idoso e o suporte da família, além dos profissionais de saúde.

\section{Conclusão}

○ perfil sociodemográfico da população pesquisada é caracterizado por predominância feminina, de cor branca, de 60 a 69 anos de idade, vive com o parceiro, é aposentada e com renda familiar de 1 a 2 salários mínimos.

Os principais fatores de risco levantados para a DAC foram: hipertensão arterial, sedentarismo, DM, tabagismo, etilismo e dislipidemia. 
Quanto ao suporte familiar destinado aos idosos, contatou-se que o convívio familiar é considerado ótimo, seguido do conceito bom, e a maioria é acompanhada em seu estado de saúde. Por sua vez, a cooperação quanto à alimentação, recursos financeiros e medicação é baixa (a maioria não recebe colaboração). No quesito exercício físico, a situação é ainda pior, pois além do alto sedentarismo a maioria dos idosos não recebe nenhum apoio familiar.

O estudo aponta a necessidade de ações de promoção da saúde e prevenção de agravos ao idoso que podem reduzir sua dependência de cuidados, de ajuda na questão econômica, material e outras. A família deve oferecer apoio ao seu idoso, que por vezes precisa ter a atenção do profissional de saúde, em especial do enfermeiro.

Os serviços e profissionais de saúde precisam criar estratégias que mostrem a responsabilidade familiar sobre seu idoso. E o Estado tem o importante papel de criar políticas públicas de suporte ao idoso, quando a família não seja capaz de fazê-lo.

\section{REFERÊNCIAS}

1.Instituto Brasileiro de Geografia e Estatística. Projeção da população do Brasil, 2013. [on-line] [citado em 05 out 2014]. Disponível em: http://www.ibge.gov.br/apps/ populacao/projecao/index.html

2.World Health Organization. Active ageing: a police framework. A contribution of the World Health Organization to the second United Nations World Assembly on Aging. Madrid (Sp): WHO; 2002.

3.Mendes MRSSB, Gusmão JL, Faro ACM, Leite RCBO. A situação social do idoso no Brasil: uma breve consideração. Acta Paul Enferm. 2005;18:422-6.

4.Ministério da Saúde (Br). Secretaria da Atenção à Saúde. Cadernos de Atenção Básica. Envelhecimento e saúde da pessoa idosa. Brasília (DF): Editora MS; 2007. 5.Silva SS, Caritá EC, Morais ERED. Fatores de risco para doença arterial coronariana em idosos: análise por enfermeiros utilizando ferramenta computacional. Esc Anna Nery. 2010; 14:797-802.

6. Oliveira AMS, Menezes TMO. A enfermeira no cuidado ao idoso na estratégia saúde da família:

sentidos do vivido. Rev enferm UERJ. 2014; 22:513-8.

7.DATASUS. Sistema de Informações Hospitalares -SIH: Ministério da Saúde. 2013. [citado em 18 out 2013]. Disponível em: http://tabnet.datasus.gov.br/cgi/tabcgi. exe?sih/cnv/qiuf.def.

8.Silveira RE, Santos AS, Sousa MC, Monteiro TSA. Gastos relacionados a hospitalizações de idosos no Brasil: perspectivas de uma década. Einsten. 2013; 11:514-20. 9.Inouye K, Barham EJ, Pedrazzani ES, Pavarini SCI. Percepções de suporte familiar e qualidade de vida entre idosos segundo a vulnerabilidade social. Psicologia: Refl Crít. 2010; 23:582-92.

10.Veras RP. Experiências e tendências internacionais de modelos de cuidado para com o idoso. Ciênc saúde coletiva. 2012; 17:231-8.
11.Instituto Brasileiro de Geografia e Estatística. Síntese de Indicadores Sociais, 2011. [on-line]. [citado em $16 \mathrm{dez}$ 2011]. Disponível em: http://www.ibge.gov.br.

12.Pinho RA, Araújo MC, Ghisi GLM, Benetti M. Doença arterial coronariana, exercício físico e estresse oxidativo. Arquivo Brasileiro de Cardiologia. 2010; 94:549-55.

13.Jacinto LAT, Santos AS, Dias FA, Silveira RE, Silvano CM. Fatores de risco para doença arterial coronariana em idosos: perspectivas para a atuação da enfermagem. Nursing (São Paulo). 2012; 15:326-30.

14.Moriel G, Roscani MG, Matsubara LS, Cerqueira ATAR, Matsubara BB. Qualidade de vida em pacientes com doença ateroclerótica coronariana grave e estável. Arquivo Brasileiro de Cardiologia. 2010; 95: 691-7.

15.Moraes EN. Atenção à saúde do idoso: aspectos conceituais. Brasília (DF): Organização Pan-Americana da Saúde; 2012. [citado 05 nov 2014]. Disponível em: http:// apsredes.org/site2012/wp-content/uploads/2012/05/ Saude-do-Idoso-WEB1. pdf

16.Ferreira OGL, Maciel SC, Costa SMG, Silva AO, Moreira MAS. Envelhecimento ativo e sua relação com a independência funcional. Texto contexto - enferm. 2012; 21:513-8.

17.Tavares DMS, Ferreira PCS, Dias FA, Oliveira PB. Caracterização e distribuição espacial de homens octogenários. Rev enferm UERJ. 2014; 22:558-64.

18.Lakatos EM, Marconi MA. Fundamentos da metodologia científica. 7aㅡ ed. São Paulo: Editora Atlas; 2010.

19.Icaza MC, Albala C. Projeto SABE. Minimental State Examination (MMSE) del estudio de dementia en Chile: análisis estatístico. Brasília (DF): OPAS; 1999.

20.Ducca GFD, Silva SG, Thumé E, Santos IS, Hallal PC. Indicadores da institucionalização de idosos: estudos de casos e controles. Rev Saude Publica. 2012; 46:147-53. 21.Pilger C, Menon MH, Mathias TAF. Características sociodemográficas e de saúde de idosos: contribuições para os serviços de saúde. Rev Latino-Am Enfermagem. [Scielo-Scientific Electronic Library Online]. 2011; [citado em 18 mar 2014]. 19(5):09 telas. Disponível em: http://www.scielo.br/pdf/rlae/v19n5/pt_22.pdf

22.Victor JF, Ximenes LB, Almeida PC, Vasconcelos FF. Perfil sociodemográfico e clínico de idosos atendidos em Unidade Básica de Saúde da Família. Acta Paul Enferm. 2009; 22:49-54.

23.Andrade W, Araújo A, Campos K. Estudo descritivo sobre a fragilidade de idosos assistidos em uma unidade de saúde da família. Rev Enferm Centro-Oeste Mineiro. 2012; [citado em 12 out 2014] Disponível em: http:// seer.ufsj.edu.br/index.php/recom/article/view/111/237 24.Ferreira CC, Peixoto MRG, Barbosa MA, Silveira EA. Prevalência de fatores de risco cardiovascular em idosos usuários do Sistema Único de Saúde de Goiânia. Arq Bras Cardiol. 2010; 95:621-8.

25.Ferro CRC, Oliveira DC, Guerra FFG, Lucena AJ, Nunes FP, Ortiz ST et al. A prevalência e fatores de risco na associação entre doença arterial coronariana e aneurisma de aorta. Arq Bras Cardiol. 2007; 88:40-4. 26. Ribeiro PCC, Neri AL, Cupertino APFB, Yassuda MS. Variabilidade no envelhecimento ativo segundo gênero, idade e saúde. Psicol estud. 2009; 14:501-9. 
27.Lemos KF, Davis R, Moraes MA, Azzolin K. Prevalência de fatores de risco para síndrome coronariana aguda em pacientes atendidos em uma emergência. Rev Gaúcha Enferm. 2010; 31:129-35

28. Nogueira AMT, Temóteo TL, Carvalho CMRG, Carvalho AMO, Borges MESMM, Luz MHBA, Teles JBM. Estudo multidimensional de idosos diabéticos atendidos em ambulatório do Sistema Único de Saúde. Rev enferm UERJ. 2010; 18:25-31.

29. Oliveira DC, Brito Junior FS, Fernandes RW. A. Doença arterial coronariana subclínica em pacientes com Diabetes Mellitus tipo 1 em hemodiálise. Arq Bras Cardiol. 2009; 93:15-21.

30.Melo Neto JS, Mendes AP, Aragão IG, Alves SA, Corrêa PR, Romano EM. Perfil dos pacientes atendidos no setor de fisioterapia cardiorrespiratória de uma clínica escola de São José do Rio Preto - SP. Arq Ciênc Saúde. 2012; 19:108-13.
31.Lima CA, Tocantins FR. Necessidades de saúde do idoso: perspectivas para a enfermagem. Rev Bras Enferm. 2009; 62:367-73.

32.Martins JJ, Pereira Nascimento ER, Erdmann AL, Candemil MC, Belaver GM. O cuidado no contexto domiciliar: o discurso de idosos/familiares e profissionais. Rev enferm UERJ. 2009; 17:556-62.

33. Victor JF, Ximenes LB, Almeida PC, Vasconcelos FF. Perfil sociodemográfico e clínico de idosos atendidos em unidade básica de saúde da família. Acta Paul Enferm. 2009; 22:49-54.

34.Nogueira IC, Santos ZMSA, Mont'Alverne DGB, Martins ABT, Magalhães CBA. Efeitos do exercício físico no controle da hipertensão arterial em idosos: uma revisão sistemática. Rev Bras de Geriatr Geront. 2012; 15:587-601. 35.Silvano CM, Contim D, Santos AS, Gonçalves JRL. O fenômeno da polifarmácia no idoso frágil. Rev pesq cuid fundam. 2012; 4:2995-3005. 